\title{
APPLICATIONS OF NANOTECHNOLOGY IN DIABETES
}

\author{
Dasari Anusha ${ }^{1 *}$, Karumanchi Pradeep ${ }^{2}$ \\ *1,2 B. tech final year, Dept. of Electronics and Communication Engineering, Jawaharlal Nehru Technological \\ University, Kakinada, A.P, India \\ *1Email: anusha.dasari94@gmail.com, ${ }^{2}$ Email: karumanchi.pradeep81@gmail.com
}

*Corresponding Author: -

Email: anusha.dasari94@gmail.com

\begin{abstract}
: -
Nanotechnology offers sensing technologies that provide more accurate and timely medical information for diagnosing disease, and miniature devices that can administer treatment automatically if required. Some tests such as diabetes blood sugar levels require patients to administer the test themselves to avoid the risk of their blood glucose falling to dangerous levels. Certain users such as children and the elderly may not be able to perform the test properly, timely or without considerable pain. Nanotechnology can now offers new implantable and/or wearable sensing technologies that provide continuous and extremely accurate medical information.The purpose of this review is to throw more light on the recent advances and impact of nanotechnology on biomedical sciences to cure diabetes.
\end{abstract}

Keywords: Diabetes, Nanotechnology, Insulin, Pancreas, Diabetes mellitus, nanomedicine.

\section{(ㄷ) (\$) (1)}




\section{INTRODUCTION}

The prevalence of diabetes is rapidly rising all over the globe at an alarming rate. Over the past $30 \mathrm{yr}$, the status of diabetes has changed from being considered as a mild disorder of the elderly to one of the major causes of morbidity and mortality affecting the youth and middle-aged people. It is important to note that the rise in prevalence is seen in all six inhabited continents of the globe. Type 2 diabetes represents approximately $90 \%$ of individuals with diabetes in the United States, while most of the remainder has type 1 diabetes. According to statistics from the Centre for Disease Control (CDC) diabetes is the sixth leading cause of death due to disease in the U.S., and the third leading cause among some ethnic populations.

The application of nanotechnology to medicine is called nanomedicine, it is defined as: "Research and technology development at the atomic, molecular and macromolecular levels in the length scale of approximately $1-100$ nanometer range, to provide a fundamental understanding of phenomena and materials at the nanoscale and to create and use structures, devices and systems that have novel properties and functions because of their small and/or intermediate size." The size domains of components involved with nanotechnology are similar to

that of biological structures. For example, a quantum dot is about the same size as a small protein $(<10 \mathrm{~nm})$ and drugcarrying nanostructures are the same size as some viruses $(<100 \mathrm{~nm})$. Because of this similarity in scale and certain functional properties, nanotechnology is a natural progression of many areas of health-related research such as synthetic and hybrid nanostructures that can sense and repair biological lesions and damages just as biological nanostructures (e.g., white-blood cells and wound-healing molecules).

\section{NANOTECHNOLOGY}

\section{A. Origins of Nanotechnology}

Nanotechnology is the study and manipulation of matter at the atomic and molecular level, this can be working with sizes from between 1 to 100 nanometres $(1 \mathrm{~nm}=10-9 \mathrm{~m})$. While the concepts of Nanotechnology were being explored from the early 60s Nanotechnology itself did not get started until later in the early 1980s with the beginnings of cluster science and the invention of the scanning tunneling microscope (STM) allowing scientists a feasible method of exploration into the world of atoms and molecules. A few years later the discovery of the fullerenes (e.g. buckminsterfullerene or C60) and carbon nanotubes allowed the researchers to take nanotechnology further and begin to engineer their own devices for varying purposes. In 2000 the US founded the National Nanotechnology Initiative in order to better co-ordinate Federal research and development. Nanomedicine is the application of Nanotechnology in medicine

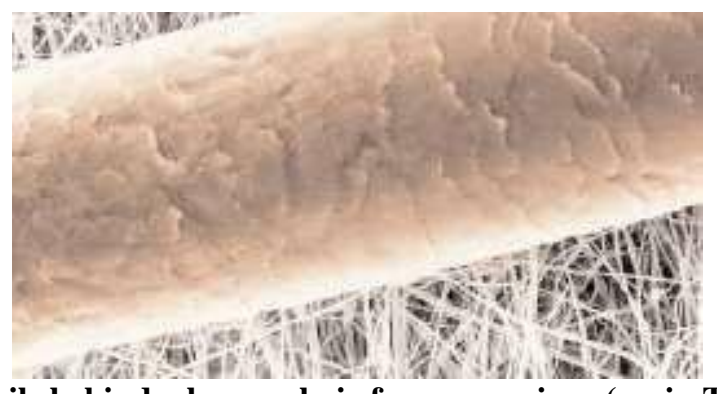

Fig 1. Nanofabrils behind a human hair for comparison (espin Technologies)

\section{B. Drug Delivery}

Nanoparticles are very small and as such will not be cleared from the body where other larger particles would have been. These small particles are taken up into the cells because of their size, and because of their shape many of them are well designed to carry substances within themselves (see figure 1- buckminsterfullerene). Currently delivery mechanisms are being developed that could allow the particles through the cell membrane and into the cytoplasm. These mechanisms will have to be very efficient as many drugs need to be within a cell before they can successfully treat a disease.

A method being explored for the distribution of drugs is 'triggered response'. This could help regulate drug release as the drug would only be activated if it came into contact with a certain 'trigger', better regulated drug release would allow for greater efficiency, currently over $\$ 65$ billion is wasted each year due to the poor precision of drugs, and eliminate the problem of tissue damage due to the quick release of a drug in one location. Poor biodistribution affects normal tissues through the widespread distribution but because of the small size of the Nanoparticles the drug is released more gradually, this also prevents the drug being cleared from the body too quickly and thereby reduces the high dosages some patients have to use.

\section{Nanoparticle Targeting}

By tracking Nanoparticles around the body (e.g. by using luminescent tags) scientists can create images of the human body that allow doctors to find out the distribution of a drug in the body or to diagnose cancer/track a tumor, for instance using a triggered response to cancerous cells. Luminescent tags are attached to proteins that penetrate cell membranes and are made of bioinert materials so they will not interfere with the body's natural processes. They can be used at various different sizes and colours so that several can be tracked at any one time, allowing for fewer light sources to be used (the current system needed as many light frequencies as cells in order to track them all). 


\section{Nanomaterials}

Nanomaterials are usually classified into two groups: fullerenes and inorganic Nanoparticles. The fullerenes are essentially graphite sheets rolled into spheres or tubes. One such material is the nanotubes; they are currently the strongest and stiffest material known and they have excellent kinetic and electrical properties. These specific properties have sparked the interest of many researchers across the world. Research at the University of California has shown carbon nanotubes to be suitable as scaffold materials for bone formation and due to their electromagnetic properties, they have been put forth as a candidate for the replacement of muscle tissue.

Nanoparticles also have a role to play in medical development, particles that have been shown to have useful properties include: colloidal gold, iron and silver particles. Colloidal gold has been shown through experimentation on rats and dogs to be an effective pain reliever when injected into a joint that has been affected by arthritis; research is being done to qualify the claims made by some scientists that Iron Nanoparticles could be used as a cheaper and healthier method of water decontamination by a redox reaction and Silver Nanoparticles are used as an antibacterial/antifungal agent in bioengineering and biotechnology but there is a effort in place to incorporate silver Nanoparticles in a range of medical devices including bone cement and wound dressings.

\section{DIABETES}

\section{A. Epidemiology}

Diabetes is a world-wide epidemic that has been made worse in recent years by the rise in obesity rates, particularly in the western world. In the UK alone there are 2.8 million people diagnosed with diabetes and a further 850,000 estimated to be unaware that they have it (Diabetes UK). Worldwide there is a much larger problem as shown in Figure 2 (the work of Wild et al. that appeared in the journal Diabetes Care in 2004)

\begin{tabular}{|c|c|c|c|c|}
\hline \multirow{2}{*}{$\begin{array}{l}\text { Rank- } \\
\text { ing }\end{array}$} & \multicolumn{2}{|l|}{2000} & \multicolumn{2}{|c|}{2030} \\
\hline & Country & $\begin{array}{l}\text { People } \\
\text { with } \\
\text { diabetes } \\
\text { (milliors) }\end{array}$ & Country & $\begin{array}{l}\text { People } \\
\text { with } \\
\text { diabetes } \\
\text { (miltions) }\end{array}$ \\
\hline 1 & India & 31.7 & India & 79.4 \\
\hline 2 & China & 20.8 & China & 42.3 \\
\hline 3 & USA & 17.7 & USA & 30.3 \\
\hline 4 & Indonesis & 8.4 & Indonesia & 21.3 \\
\hline 5 & Japan & 6.8 & Pakistan & 13.9 \\
\hline 6 & Pakistan & 5.2 & Brazil & 11.3 \\
\hline 7 & Russian Federation & 4.6 & Bangladesh & 11.1 \\
\hline 8 & Brazil & 4.6 & Japan & 8.9 \\
\hline 9 & Italy & 4.3 & Philippines & 7.8 \\
\hline 10 & Bangladesh & 3.2 & Egypt & 6.7 \\
\hline
\end{tabular}

Fig 2. Global Prevalence of Diabetes: Estimates for the year 2000 and projections for 2030

\section{B. Pathophysiology}

Diabetes mellitus, often simply referred to as diabetes - is a group of metabolic diseases in which a person has high blood sugar, either because the body does not produce enough insulin, or because cells do not respond to the insulin that is produced. This high blood sugar produces the classical symptoms of polyuria (frequent urination), polydipsia (increased thirst) and polyphagia (increased hunger). There are three main types of diabetes:

- Type 1 diabetes: results from the body's failure to produce insulin, and presently requires the person to inject insulin

- Type 2 diabetes: results from insulin resistance, a condition in which cells fail to use insulin properly, sometimes combined with an absolute insulin deficiency.

- Gestational diabetes: is when pregnant women, who have never had diabetes before, have a high blood glucose level during pregnancy. It may precede development of type $2 \mathrm{DM}$.

Other forms of diabetes mellitus include congenital diabetes, which is due to genetic defects of insulin secretion, cystic fibrosis-related diabetes, steroid diabetes induced by high doses of glucocorticoids, and several forms of monogenic diabetes.

All forms of diabetes have been treatable since insulin became available in 1921, and type 2 diabetes may be controlled with medications. Both type 1 and 2 are chronic conditions that usually cannot be cured. Pancreas transplants have been tried with limited success in type 1 DM; gastric bypass surgery has been successful in many with morbid obesity and type 2 DM Acute complications include hypoglycemia, diabetic ketoacidosis, or nonketotic hyperosmolar coma. Serious longterm complications include cardiovascular disease, chronic renal failure, retinal damage. Adequate treatment of diabetes is thus important, as well as blood pressure control and lifestyle factors such as smoking cessation and maintaining a healthy body weight. Gestational diabetes usually resolves after delivery. Diabetes without proper treatments can cause many complications. 


\section{Type 1 diabetes}

Diabetes mellitus type 1 (Type 1 diabetes, IDDM, or, formerly, juvenile diabetes) is a form of diabetes mellitus that results from autoimmune destruction of insulin-producing beta cells of the pancreas. The subsequent lack of insulin leads to increased blood and urine glucose. The classical symptoms are polyuria (frequent urination), polydipsia (increased thirst), polyphagia (increased hunger), and weight loss.

In the long run, type 1 diabetes is generally fatal unless treated with insulin. Injection is the most common method of administering insulin; insulin pumps and inhaled insulin have been available at various times. Pancreatic transplants and pancreatic islet cell transplantation have been used to treat type 1 diabetes; however, pancreatic islet cell transplantation is still viewed as experimental, although utilization of the procedure is growing. Most people who develop type 1 are otherwise healthy. Although the cause of type 1 diabetes is still not fully understood it is believed to be of immunological origin.

Type 1 can be distinguished from type 2 diabetes via a C-peptide assay, which measures endogenous insulin production. Type 1 treatment must be continued indefinitely in all cases. Treatment is not intended to significantly impair normal activities, and can be done adequately if sufficient patient training, awareness, appropriate care, discipline in testing and dosing of insulin is taken. However, treatment remains quite burdensome for many people. Complications may be associated with both low blood sugar and high blood sugar, both largely due to the non- physiological manner in which insulin is replaced. Low blood sugar may lead to seizures or episodes of unconsciousness and requires emergency treatment. High blood sugar may lead to increased fatigue and can also result in long term damage to organs.

\section{Type 2 diabetes}

Diabetes mellitus type 2 - formerly non-insulin-dependent diabetes mellitus (NIDDM) or adult-onset diabetes - is a metabolic disorder that is characterized by high blood glucose in the context of insulin resistance and relative insulin deficiency. Diabetes is often initially managed by increasing exercise and dietary modification. If the condition progresses, medications may be needed. Often affecting the obese, diabetes requires patients to routinely check their blood sugar.

Unlike type 1 diabetes, there is very little tendency toward ketoacidosis though it is not unheard of. One effect that can occur is nonketonic hyperglycemia. Long-term complications from high blood sugar can include increased risk of heart attacks, strokes, amputation, and kidney failure. For extreme cases, circulation of limbs is affected, potentially requiring amputation. Loss of hearing, eyesight, and cognitive ability has also been linked to this condition

\section{Gestational diabetes}

Gestational diabetes mellitus (GDM) resembles type 2 diabetes in several respects, involving a combination of relatively inadequate insulin secretion and responsiveness. It occurs in about $2 \%-5 \%$ of all pregnancies and may improve or disappear after delivery. Gestational diabetes is fully treatable but requires careful medical supervision throughout the pregnancy. About $20 \%-50 \%$ of affected women develop type 2 diabetes later in life.

Even though it may be transient, untreated gestational diabetes can damage the health of the fetus or mother. Risks to the baby include macrosomia (high birth weight), congenital cardiac and central nervous system anomalies, and skeletal muscle malformations. Increased fetal insulin may inhibit fetal surfactant production and cause respiratory distress syndrome. Hyperbilirubinemia may result from red blood cell destruction. In severe cases, perinatal death may occur, most commonly as a result of poor placental perfusion due to vascular impairment. Labor induction may be indicated with decreased placental function. A cesarean section may be performed if there is marked fetal distress or an increased risk of injury associated with macrosomia, such as shoulder dystocia

Diabetes mellitus is a metabolic disorder resulting from a defect in insulin secretion, insulin action or both. Insulin deficiency in turn leads to chronic hyperglycaemia with disturbances of carbohydrate, fat and protein metabolism. It is the most common endocrine disorder and by the year 2012, it is estimated that more than 200 million people worldwide will have DM and 300 million will subsequently have the severe diabetic complication such as retinopathy, neuropathy, nephropathy, cardiovascular complication and ulceration. Most complications of diabetes are due to either acute metabolic disturbances or chronic tissue damage. Thus, diabetes covers a wide range of heterogeneous disease. Preventative methods for diabetes are as yet poorly developed. More progress has been made with potentially curative surgery. However, at present the vast majority of people with diabetes require long-term management of established disease.

\section{PREDICTORS, RISK FACTORS AND SYMPTOMS}

There are no sure predictors of either type of diabetes and there is at the moment no way of preventing type 1 diabetes; however, there are many risk factors that can be taken into account for type 2 diabetes. These include: age, family history of type 2 diabetes, weight, high blood pressure and impaired glucose tolerance.

In type 1 diabetes the symptoms can come on very rapidly as no glucose is being produced, however in type 2 diabetes some of the insulin is still working so the onset is much slower, a person may live with undiagnosed diabetes for up to 10 years. The symptoms of both types of diabetes are the same: more frequent urination (due to the body's need to rid itself of the excess glucose in the blood), thirst (due to excess urination), extreme tiredness (lack of glucose in the cell for fuel), unexplained weight loss (an attempt of the body to get more glucose to the cells by breaking down fatty deposits, genital itching or regular episodes of thrush (excess glucose in urine encourages bacterial growth), blurred vision (glucose deposits in the lens of the eye) and slow healing cuts and wounds (bacteria breeding in flesh wounds due to high glucose content in the blood). 


\section{NANOMEDICINE APPLICATION IN GLUCOSE MONITORING}

The major problems with conventional finger-prick capillary blood glucose self-monitoring are widely accepted. It is painful (leading to non-compliance) and cannot be performed when the patient is sleeping or driving a motor vehicle (times when the patient is especially vulnerable to hypoglycaemia) and, because it is intermittent, it can miss dangerous fluctuations in blood glucose concentrations between tests. Currently in market several implanted needle- type enzyme electrodes or microdialysis probes are available for continuous glucose monitoring but those are limited impaired responses and unpredictable signal drift in vivo, and also need calibration against capillary glucose tests and contributes to sensor inaccuracies. The repeated insertion of the sensor probe is also semi-invasive. This conventional problem got solution by the application of nanotechnology in medicine.

\section{A. Glucose Nanosensors}

Improved nanotechnique for in vivo glucose monitoring is a 'smart tattoo' composed of glucose-responsive, fluorescencebased nanosensors implanted into the skin but interrogated from outside the body, thus gives non- invasive measurements. In this method sensors that use fluorescence for detecting analyte changes have some advantages compared to the more usual implanted electrochemical electrodes, as they should not be susceptible to electroactive tissue interfearence that contribute to the instability of present sensors, and because Near infrared (NIR) light with a wavelength above about 600 $\mathrm{nm}$ passes through several centimeters of tissue, allowing implantation and non-invasive measurement at the body surface. Currently a number of biological or artificial receptors for glucose have been described, which can transduce glucose concentrations into changes in fluorescence, including lectins (plant lectin concanavalin-A), enzymes (hexokinase), bacterial binding proteins(Glucose/Galactose-Binding Protein (GBP) and boronic acid derivatives, and which might be engineered as nanosensors.

\section{B. The layer-by-layer (LBL) technique}

Glucose sensors are encapsulated in a form that can be implanted in the body and yet maintain functionality i.e, avoiding degradation, denaturation, leakage and foreign body reactions, while retaining glucose access and detectable signal change. An example of a technology that may be appropriate for this is electrostatic layer-by-layer (LBL) nanoassembly of capsules composed of alternating layers of positively and negatively charged polymers, thereby building up a very thinfilm with tunable permeability and controlled biocompatibility. A typical thickness is approximately $10 \mathrm{~nm}$ for six bilayers. An early use of such nanofilms has been in the construction of microvesicles for glucose sensing. These might eventually be implanted in the dermis or subcutaneous tissue as a 'smart tattoo', with the semipermeable capsules allowing entry of glucose from the interstitial fluid but containing and protecting the sensor material. If the sensing mechanism involves a near infrared (NIR)-based fluorescence assay, the glucose sensors can be excited and the fluorescence emission interrogated from outside the body, since NIR light passes through several centimeters of tissue. Therefore, this might be the basis for a noninvasive glucose- sensing technology. Among the strategies for making microvesicles by the LBL technique are sequential absorption of polyelectrolytes around crystals of a glucose-sensing enzyme such as glucose oxidase, or absorption of a glucose-recognition molecule such as glucose oxidase, apo-glucose oxidase (the enzyme without the prosthetic group), concanavalin A or a glucose-binding protein (GBP) onto a artificial template (e.g.,Calcium carbonate), followed by stepwise addition of the charged polypeptides (e.g., polyl- lysine and polyl-glutamic acid). The template is then dissolved with ethylenediaminetetraacetic acid (EDTA), leaving a hollow microcapsule containing the sensor.

\section{Carbon nanotubes}

Carbon nanotubes discovered in 1991 are tubular structures like a sheet of graphite rolled into a cylinder capped at one or both ends by a buckyball. Nanotubes can be Single Walled Carbon Nanotube (SWCNT) or Multiwalled Carbon Nanotube (MWCNT) in concentric fashion. The microphysiometer is built from multiwalled carbon nanotubes, which are like several flat sheets of carbon atoms stacked and rolled into very small tubes. Which are like several flat sheets of carbon atoms stacked and rolled into very small tubes. The nanotubes are electrically conductive and the concentration of insulin in the chamber can be directly related to the current at the electrode and the nanotubes operate reliably at $\mathrm{pH}$ levels characteristic of living cells.Current detection methods measure insulin production at intervals by periodically collecting small samples and measuring their insulin levels. The new sensor detects insulin levels continuously by measuring the transfer of electrons produced when insulin molecules oxidize in the presence of glucose. When the cells produce more insulin molecules, the current in the sensor increases and vice versa, allowing monitoring insulin concentrations in real time. Single-Walled Carbon Nanotubes (SWCNTs) fluorescence in the NIR spectral region and since they also suffer no photobleaching, SWCNTs are thus particularly suitable as fluorophore probes in glucose sensors designed for eventual in vivo use. SWCNTs have been employed in a fluorescence-based competitive glucose sensing strategy where dextran is bound to the carbon nanotubes, and binding of concanavalin A or apo-glucose oxidase to the dextran-SWCNT attenuates the fluorescence, which is reversed by the addition of glucose. Apo-glucose oxidase has also been covalently attached to polyvinyl alcohol to make a glucose responsive hydrogel that can be monitored by the fluorescence of SWCNT embedded in the hydrogel

\section{Quantum dots}

Quantum Dots (QDs) can be used for biomedical purposes as a diagnostic as well as therapeutic tool. These arenanosized $(2-10 \mathrm{~nm})$ semiconductor crystals, such as cadmium selenide, coated with a shell, such as zinc sulfide. QDs have been used as a fluorescent probe in several biosensor applications, often using As Fluorescence- Resonance Energy Transfer 
(FRET), because they display high-intensity fluorescence that is excitable over a broad range of wavelengths, but have an emission wavelength that is dependent on the particle size. For example, based a glucose sensor on FRET between QDs as a fluorescence donor and gold nanoparticles as an acceptor the, glucose displaces concanavalin A-labeled QDs from gold-labeled cyclodextrin, thereby, reducing FRET and increasing fluorescence.

\section{E. Microphysiometer}

The microphysiometer is built from multiwalled carbon nanotubes, which are like several flat sheets of carbon atoms stacked and rolled into very small tubes. It can be used to detect and monitor the response of cells to a variety of chemical substances, especially ligands for specific plasma membrane receptors. The nanotubes are electrically conductive and the concentration of insulin in the chamber can be directly related to the current at the electrode and the nanotubes operate reliably at $\mathrm{pH}$ levels characteristic of living cells. Current detection methods measure insulin production at intervals by periodically collecting small samples and measuring their insulin levels. The new sensor detects insulin levels continuously by measuring the transfer of electrons produced when insulin molecules oxidize in the presence of glucose. When the cells produce more insulin molecules, the current in the sensor increases and vice versa, allowing monitoring insulin concentrations in real time.

\section{F. Implantable sensor}

An implantable sensor capable of long-term monitoring of tissue glucose concentrations by wireless telemetry has been developed for eventual application in people with diabetes. The implantable sensor is designed to give diabetes patients an alternative to finger-sticking or short-term glucose sensors, as well as limit dangerous glucose level fluctuations known as "glucose excursions". Use of polyethylene glycol beads coated with fluorescent molecules to monitor diabetes blood sugar levels is very effective in this method the beads are injected under the skin and stay in the interstitial fluid. When glucose in the interstitial fluid drops to dangerous levels, glucose displaces the fluorescent molecules and creates a glow. This glow is seen on a tattoo placed on the arm. Sensor microchips are also being developed to continuously monitor key body parameters including pulse, temperature and blood glucose. A chip would be implanted under the skin and transmit a signal that could be monitored continuously.

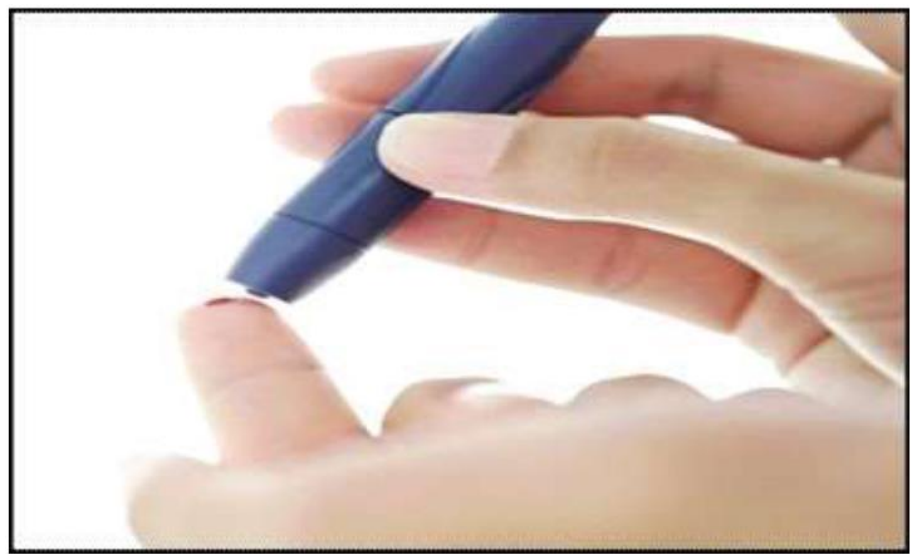

Fig 3. The "pin-prick" glucose test could be replaced with more accurate and convenient methods using Nanotechnology

\section{NANOMEDICINE IN MANAGEMENT OF DIABETES}

Worldwide 285 million peoples are suffering with a pervasive,chronic and often insidious diabetes is caused by inability of the pancreas to control the blood glucose concentration. The preferred approach of insulin intake since the past decades is via subcutaneous route, which, nonetheless, often fails to mimic the glucose homeostasis observed in normal subjects because in this approach insulin delivered to the peripheral circulation rather than to the portal circulation and directly into the liver, which is the physiological route in normal individuals. Furthermore, multiple daily injections of insulin referred for poor patient compliance are associated with subcutaneous route treatment. Therefore, many studies were done to find out the better and safer route of insulin administration, in this regards application of nanotechnology in medicine revealed a solution to overcome this problem.

\section{A. Oral insulin}

In diabetic patients' oral administration of insulin can be beneficial not only to alleviate the pain and trauma caused by injections, but it can mimic the physiological fate of insulin as well. However, oral administration of protein drugs, such as insulin, encounters difficulties with low $\mathrm{pH}$ of gastric medium in the stomach and its digestive enzymes as well as intestinal epithelium is a major barrier to the absorption of molecular weight hydrophilic macromolecules (e.g proteins, polysaccharides and nucleic acids) before it reaches the target cell for it specific action. Therefore, attentions have been given to improving the paracellular delivery of hydrophilic macromolecules with the application of nanotechnology in diabetic research. The nanomedicine technologies that may be employed for oral insulin delivery include prodrugs (insulin-polymer conjugation), micelles, liposomes, solid lipid nanoparticles (NPs) and NPs of biodegradable polymers. The prodrug technology that is used most often for drug formulation is PEGylation (i.e., drug conjugation to Polyethylene 
Glycol [PEG]) for enhanced solubility, permeability and stability. Insulin-PEG prodrugs have shown great advantages in oral delivery.Scientists developed a system of bilesalt and fattyacid mixed micelles and found that those micelles containing $30 \mathrm{mM}$ sodium glycocholate and $40 \mathrm{mM}$ linoleic acid significantly improved enteral insulin absorption. Unfortunately, micelles did not seem to be ideal for delivery of hydrophilic drugs. Instead, liposomes can have much better performance for oral insulin delivery. This liposomal delivery system containing glycocholate as an enzyme inhibitor and permeat ion enhancer has been developed recently for oral insulin delivery, which showed better protect ion of insulin against enzymatic degradation by pepsin, trypsin and achymotrypsin. NPs of USFDA approved biodegradable polymers, such as poly (lacticcoglycolic acid) (PLGA) and polycaprolactone, were also investigated for oral insulin delivery. However, those NPs may not be ideal for delivery of hydrophilic drugs. Nevertheless, how it can be applied to oral delivery for hydrophilic drugs such as insulin is still a challenge. Therefore, attention has been given to improving the paracellular transport of hydrophilic drugs. A variety of intestinal permeation enhancers including chitosan (CS) have been used for the assistance of the absorption of hydrophilic macromolecules. Therefore, a carrier system is needed to protect protein drugs from the harsh environment in the stomach and small intestine, if given orally. Additionally, CS nanoparticles (NPs) enhanced the intestinal absorption of protein molecules to a greater extent than aqueous solutions of CS in vivo. The insulin loaded NPs coated with muco adhesive CS may prolong their residence in the small intestine, infiltrate into the mucus layer and subsequently mediate transiently opening the tight junctions between epithelial cells while becoming unstable and broken apart due to their $\mathrm{pH}$ sensitivity and/or degradability. The insulin released from the broken-apart NPs could then permeate through the paracellular pathway to the bloodstream, its ultimate destination.

\section{B. Polymeric Nanoparticles}

Polymeric nanoparticles have been used as carriers of insulin. The use of biodegradable polymeric nanoparticles for controlled drug delivery has shown significant therapeutic potential. These are biodegradable polymers, with the polymer-insulin matrix surrounded by the nanoporous membrane containing grafted glucose oxidase. A rise in blood glucose level triggers a change in the surrounding nanoporous membrane, resulting in biodegradation and subsequent insulin delivery. The glucose/glucose oxidase reaction causes a lowering of the $\mathrm{pH}$ in the delivery system's microenvironment. This can cause an increase in the swelling of the polymer system, leading to an increased release of insulin. The polymer systems investigated for such applications include copolymers such as $N, N$-dimethylaminoethyl methacrylate and polyacrylamide. This "molecular gate" system is composed of an insulin reservoir and a delivery ratecontrolling membrane made of poly [methacrylic acid-g-poly (ethylene glycol)] copolymer. The polymer swells in size at normal body $\mathrm{pH}(\mathrm{pH}=7.4)$ and closes the gates. It shrinks at low $\mathrm{pH}(\mathrm{pH}$

$=4$ ) when the blood glucose level increases, thus opening the gates and releasing the insulin from the nanoparticles. These systems release insulin by swelling caused due to changes in blood $\mathrm{pH}$. The control of the insulin delivery depends on the size of the gates, the concentration of insulin, and the rate of gates' opening or closing (response rate). These selfcontained polymeric delivery systems are still under research, whereas the delivery of oral insulin with polymeric nanoparticles has progressed to a greater extent in the recent years.

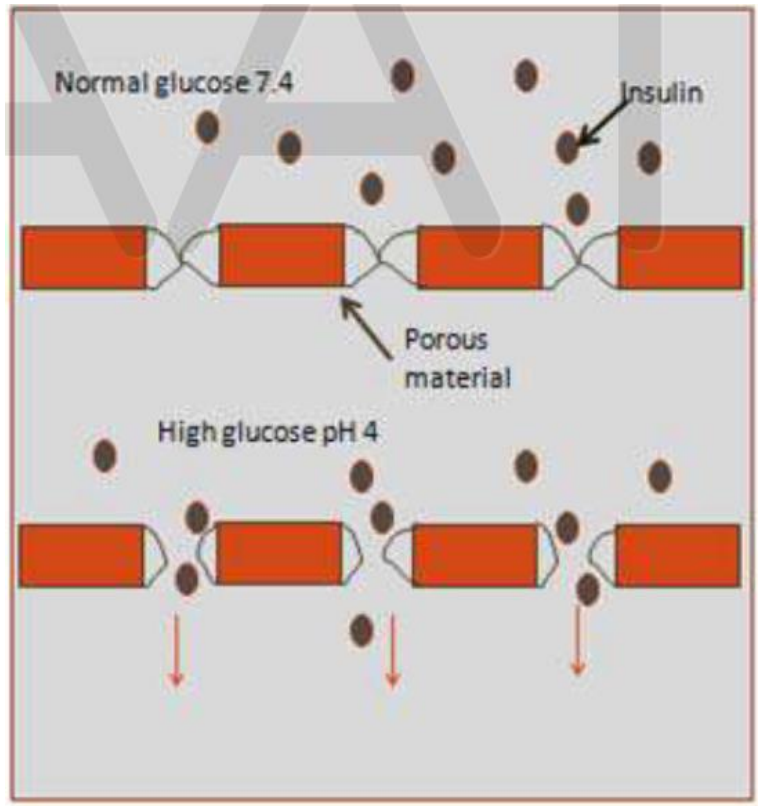

Fig 4: Schematic of polymeric nanoparticles with pH-sensitive molecular gates for controlled insulin release triggered by the presence of glucose in blood

\section{Oral Insulin Administration by Using Polysaccharides and Polymeric Nanoparticles}

Polysaccharides are natural biodegradable hydrophilic polymers, which exhibit enzymatic degradation behavior and good biocompatibility ${ }^{[16]}$. The development of improved oral insulin administration is very essential for the treatment of diabetes mellitus to overcome the problem of daily subcutaneous injections. Insulin, when administered orally, undergoes degradation in the stomach due to gastric enzymes. Therefore, insulin should be enveloped in a matrix like system to 
protect it from gastric enzymes. This can be achieved by encapsulating the insulin molecules in polymeric nanoparticles. In one such study, calcium phosphate-poly (ethylene glycol)-insulin combination was combined with casein (a milk protein). The casein coating protects the insulin from the gastric enzymes. Due to casein's mucoadhesive property, the formulation remained concentrated in the small intestine for a longer period, resulting in slower absorption and longer availability in the bloodstream.

\section{Insulin Delivery through Inhalable Nanoparticles}

Inhalable, polymeric nanoparticle-based drug delivery systems have been tried earlier for the treatment of tuberculosis. Such approaches can be directed toward insulin delivery through inhalable nanoparticles. Insulin molecules can be encapsulated within the nanoparticles and can be administered into the lungs by inhaling the dry powder formulation of insulin. The nanoparticles should be small enough to avoid clogging up the lungs but large enough to avoid 414 being exhaled. Such a method of administration allows the direct delivery of insulin molecules to the bloodstream without undergoing degradation. A few studies have been done to test the potential use of ceramic nanoparticles (calcium phosphate) as drug delivery agents. Porous hydroxyapatite nanoparticles have also been tested for the intestinal delivery of insulin. Preclinical studies in guinea pig lungs with insulin-loaded poly (lactide-co-glycolide) nanospheres demonstrated a significant reduction in blood glucose level with a prolonged effect over 48 hours when compared with insulin solution. Insulin-loaded poly (butyl cyanoacrylate) nanoparticles when delivered to the lungs of rats were shown to extend the duration of hypoglycemic effect over 20 hours when compared with pulmonary administration of insulin solution. The major factors limiting the bioavailability of nasally administered insulin include poor permeability across the mucosal membrane and rapid mucociliary clearance mechanism that removes the non-mucoadhesive formulations from the absorption site. To overcome these limitations, mucoadhesive nanoparticles made of chitosan/tripolyphosphate and starches have been evaluated. These nanoparticles showed good insulin-loading capacity, providing the release of $75 \%$ to $80 \%$ insulin within 15 minutes after administration

\section{E. Nanopumps}

The nanopump is a powerful device and has many possible applications in the medical field. The first application of the pump, introduced by Debiotech for insulin delivery. The pump injects insulin to the patient's body in a constant rate, balancing the amount of sugars in his or her blood. The pump can also administer small drug doses over a long period of time.

\section{F. Nanoparticle's anti oxidative role in diabetes}

Oxidative stress plays a foremost role in etiology of several diabetic complications. Major problem with diabetes patients is delayed healing of wound and still challenging its complete cure. It is a complex programmed sequence of cellular and molecular processes. Healing impairment is characterized by delayed cellular infiltration and granulation tissue formation, reduced angiogenesis, decreased collagen, and its organization. The mechanism of this alteration is thought to result from production of high level of ROS production that leads to premature apoptosis of inflammatory cells, which in turn impairs keratinocyte endothelial cells, fibroblasts, and collagen metabolism. Some of the nanoparticle now a day are preparing in a manner that act as free radical scavenger. Cerium oxide $(\mathrm{CeO} 2)$ plays major active role due to its excellent free radical scavenging potentials. This metal oxide is monodispersed particle with single crystal and few twin boundaries with expanded lattice parameter. Cerium atom characterized by both +4 and +3 oxidation states. This dual oxidation state means that these nanoparticles have oxygen vacancies. The loss of oxygen and the reduction of $\mathrm{Ce} 4+$ to $\mathrm{Ce} 3+$ is accompanied by creation of an oxygen vacancy. This property enhances $\mathrm{CeO} 2$ nanoparticle attractive for wound healing process followed by scavenging properties. Yttrium oxide (Y2O3) is now a day considered most significant due to its highest free energy of oxide formation from elemental yttrium among known metal oxides. It is characterized by only small changes from stoichiometry under normal conditions of temperature and pressure and by atmospheric absorption of $\mathrm{H} 2 \mathrm{O}$ and $\mathrm{CO} 2$ These groups of nanoparticles are relatively nontoxic to neutrophils and macrophages, where $\mathrm{CeO} 2$ and Y2O3 particles protect cells from death due to oxidative stress. Cerium and yttrium oxide nanoparticles are able to rescue cells from oxidative stress-induced cell death in a manner that appears to be dependent upon the structure of the particle but independent of its size within the range of 6-1000 $\mathrm{nm}$. This might be useful for the diabetic wound healing. Literature shows three alternative explanations for the cerium oxide and yttrium oxide particles protection against oxidative stress.

(a) They may act as direct antioxidants, block ROS production, which inhibit programmed cell death pathway

(b) They may directly cause a low level of ROS production, which rapidly induces a ROS defense system before the glutamate-induced cell death program is complete and

(c) The latter is a form of preconditioning that could be caused by the exposure of cells to particulate material known to induce low levels of ROS. Nanoparticles made of other metal oxides were also considered for its potential scavenger behavior. These included particles aluminium oxide (Al2O3), commonly known as alumina and silver nitrate. Silver nitrate has been used in the clinical setting as an antimicrobial agent for the treatment of treatment of chronic wounds. It is effective against a broad range of aerobic, anaerobic, Gram-negative and Gram-positive bacteria, yeast, filamentous fungi and viruses. It has been reported a novel $\mathrm{Ag}+$ loaded zirconium phosphate nanoparticle plays crucial role in diabetic wound healing. The gold nanoparticles (AuNPs), are known for their tremendous applications in the field of theapeutics and diagonosis. AuNPs are emerging nanomedicine which is renowned for its promising therapeutic possibilities, due to its significant properties such as biocompatibility, high surface reactivity, resistance to oxidation and plasmon resonance. The ability of gold nanoparticles in inhibiting the lipid from peroxidation thereby preventing the ROS generation has restored the imbalances in the antioxidants. 
Scientists are also trying to create a nanorobot which would have insulin departed in inner chambers, and glucose- level sensors on the surface. When blood glucose levels increase, the sensors on the surface would record it and insulin would be released. Yet, this kind of nano-artificial pancreas is still only a theory (Fig. 5).

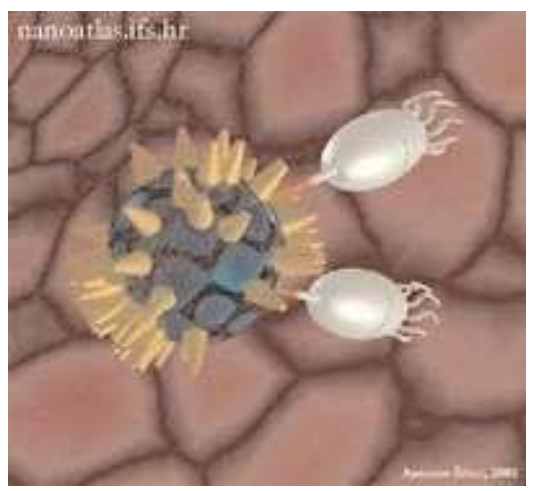

Fig 5. Nanobots killing a virus

\section{APPLICATIONS OF NANOTECHNOLOGY IN HEALTH CARE AND DISEASE}

Nanotechnology, by development of engineered nanomaterials, is a rapidly growing industry with great potential and applications in many areas, including nanomedicine. Nanomaterials (NMs) have unique properties and applications when it comes to drug delivery and imaging, and they have the potential to improve diagnostics and therapy of many human disorders, including neurodegenerative disorders, by their ability to cross the Blood Brain Barrier (BBB). The nanosize and the large surface area of nanoparticles (NPs) reflects increased reactivity, and even an inert bulk compound, such as gold, may elicit a response in humans when administered as a NMs. However, concern has been raised that the properties that make NMs unique and so useful could also be coupled to unintentional effects on human health. Thus, it is important to obtain information about the potential toxicity of NMs to discover and prevent serious unwanted human effects. The goal must be to realize the great opportunities and benefits of NMs while at the same time minimizing the risk related to their applications. Nanomedicine is a field with continuous progress, introducing novel applications in many health care areas. The underlying motivation is improvement of quality of life with economic and social benefits. Some of the most promising areas are the following $[80,81]$.

- Nanodiagnostics (molecular diagnostics, imaging using NPbased contrast materials, nanobiosensors)

- Nanopharmaceuticals (targeted drug delivery, nanotechnologybased drugs, implanted nanopumps, nanocoated stents)

- Reconstructive surgery (tissue engineering, implantation of rejection resistant artificial tissues and organs)

- Nanorobotics (vascular surgery, detection and destruction of cancer)

- Nanosurgery (nanolasers, nanosensors implanted in catheters)

- Regenerative medicine (tissue repair)

- Ultrafast DNA sequencing.

\section{POTENTIAL TOXICITY OF NANOPARTICLES}

There is as yet no reason to think that nanomaterials pose a certain toxic threat or that any possible harmful effects cannot be assessed and managed as is done for any new pharmaceuticals, diagnostics or medical materials. Nanoparticles, as a result of their extreme microscopic dimension, which gives unique advantage, have potential hazards similar to particulate matter. These particles have the potential to cause varied pathologies of respiratory, cardiovascular and gastrointestinal system.However, as there is a lack of information and research on nanomaterial toxicity, it is important to consider the potential health and safety issues. The determinant of particle toxicity are known to be the large surface area and chemical reactivity in relation to small size (and thus the ability to generate reactive oxygen species) and the ability to penetrate tissues and cells. Thus, nanoparticles are likely to be more hazardous than the same chemicals in larger form, and free particles more toxic than fixed ones. The potential cytotoxicity of QDs is an example to consider, because at high concentrations harmful effects on embryo development and cell viability and function have been recorded . Nanoparticles such as QDs most likely need to be passivated with less toxic materials to improve biocompatibility, for example with silica . Intratracheal instillation of carbon nanotube particles in mice has shown that carbon nanotubes have the potential to cause varied lung pathologies like epitheloid granuloma, interstitial inflammation, peribronchial inflammation and necrosis of lung. The toxicity produced by carbon nanotube was found to be greater than that produced by carbon black and quartz. Studies done on monkeys and rats have shown accumulation of carbon and manganese nanoparticles in the olfactory bulb through the olfactory pathway. This shows that nanoparticle mediated delivery can in future provide a means of alternate route, circumventing the blood brain barrier. However, this can also result in the inflammatory reactions in the brain which needs to be evaluated. It was observed that the nanoparticles can reach the circulation and reach different organs and systems and possibly results in toxicity. These have been studied in vitro and in animal models and the effect on human system is difficult to extrapolate from such studies. Their use in humans require further research and much needed caution. reactions in the brain which needs to be evaluated. 


\section{CONCLUSION}

This article was an attempt to review the chief scientific and technical aspects of nanomedicine in related to diabetes. However the expectation from nanotechnology in medicine are high and the potential benefits are endlessly enlisted but the safety of nanomedicine is not yet clear. Nanomedicine shows great potential for the future diabetic management and at the moment the suggested benefits in diabetic health care outweigh the possible dangers of nanoparticles use in medicine. Here we conclude that use of nanomedicine in diabetic care is in initial stage, but progress is rapid.Inspite of diabetes having many remaining problems,nanomedicine is likely to be a key technology for solving many of them and will be a core technology in diabetic research.

\section{REFERENCES}

[1].Bloodless

Diabetes

Monitoring

http://www.nano.org.uk/nanomednet/index.php?option=com_content\&task=view\&id=184\&Itemid=1

[2].Carbon Nanotube -http://en.wikipedia.org/wiki/Carbon_nanotube

[3].Causes and Risk Factors -http://www.diabetes.org.uk/Guide-to-diabetes/Introduction-todiabetes/Causes_and_Risk_Factors

[4].Nanomaterials -http://en.wikipedia.org/wiki/Nanomaterials

[5].Nanotechnology -http://en.wikipedia.org/wiki/Nanotechnology Nanotechnology and Diabetes http://www.tweetlebeetle.ca/wpcontent/uploads/2010/10/Nanotechnology-and-

[6].Diabetes.pdf

[7].Using Nanotechnology in Diabetes -http://www.online-diabetes-information.com/introduction-to-diabetes/usingnanotechnology-in-diabetes/

[8].What is Diabetes? -http://www.diabetes.org.uk/Guide-to-diabetes/Introduction-to-diabetes/What_is_diabetes/ 